\title{
Chaos of time of the Universe
}

\section{Chelnokov M.}

Department of Physics, Bauman Moscow State Technical University, Moscow, Russia;

E-mail: Chelnokov<l-chelnok@yandex.ru>;

This work states the connection between the spatial inversion and the inversion of time and the transition from one inertial reference system to another. The received date state that in the Universe there is no single direction of time and that chaos of time reigns in it. The article analyzes a number of other results. The idea of an experiment for testing of the introduced concepts is suggested.

Keywords: inversion, Universe, cosmology, reference system, space, time, Minkowski's world, events, signal, time chaos.

DOI: $10.18698 / 2309-7604-2015-1-121-130$

\section{Introduction}

In a previous work [1] the author considered the problems of mirror asymmetry, spatial inversion and non-conservation of $P$-parity. The given work is, to some extent, a continuation of the previous one and it is dedicated, first and foremost to the inversion of time.

Until now it was considered that we can speak of the single direction of time in the Universe on the whole - from the past to the future. At present, it is well known in the frame of the special theory of relativity (STR) time has different «speed» in different inertial reference systems (IRS) and can even «move» in opposite directions, and in the framework of the general theory of relativity, time slows down near gravitating bodies.

However, it did not prevent us from considering the flow of time on the whole, for example, the lifetime of the Universe since the Big Bang is estimated at 13 billion years.

Being entirely consistent and while analyzing the time in the framework of SRT, our idea of the single direction of time in the Universe loses its sense as, for example, the idea of the absolute simultaneity.

Besides it turns out that the known operation of inversion (both spatial and inversion of time) under some conditions equivalent to the operation of the transition from one IRS to another within SRT.

\section{Inversion of time}

We introduce the following reasonable, in our view, definition of different direction of time: 
Suppose that in some IRS K the event 2 occurs after the event 1, and IRS K' event 1 occurs after event 2. So we can say that in these two IRS time flows in the opposite directions.

In both of these systems, time flows from the past to the future, but what is the future for IRS $\mathrm{K}$ turns out to be the past for IRS $\mathrm{K}^{\prime}$, and the past for IRS $\mathrm{K}$ appears to be the future for IRS K'.

Suppose there are two events: Event 1 and Event 2 (or 3). Let us consider them from the point of view of IRS K and IRS K' (Figure 1). Reference system $K^{\prime}$ moves relative to the reference system $\mathrm{K}$ along coincident axes $x$ and $x^{\prime}$ to the right with velocity $\boldsymbol{V}$, or, what is the same, the reference system $\mathrm{K}$ moves relative to the reference system $K^{\prime}$ to the left with velocity $\boldsymbol{V} \boldsymbol{V}$.

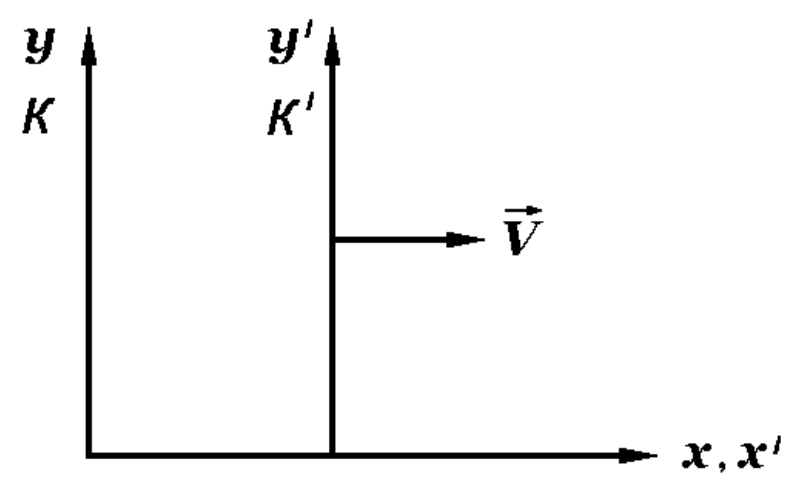

Fig. 1. Two inertial reference systems in SRT

As it is known [2,3], in the SRT have the following formulas of transformation of periods of time.

Transition IRS K' $\rightarrow$ IRS K:

$$
c \Delta t=c\left(t_{2}-t_{1}\right)=\gamma\left[c\left(t_{2}{ }^{\prime}-t_{1}{ }^{\prime}\right)+\beta\left(x_{2}{ }^{\prime}-x_{1}{ }^{\prime}\right)\right]
$$

Transition IRS $\mathrm{K} \rightarrow$ IRS K':

$$
c \Delta t^{\prime}=c\left(t_{2}^{\prime}-t_{1}^{\prime}\right)=\gamma\left[c\left(t_{2}-t_{1}\right)-\beta\left(x_{2}-x_{1}\right)\right]
$$

Here we introduce the usual symbols:

$$
\text { c-velocity of light; }
$$




$$
\begin{gathered}
\beta=\frac{V}{c} \\
\gamma=\frac{1}{\sqrt{1-\beta^{2}}}
\end{gathered}
$$

Primed quantities refer to the IRS $\mathrm{K}^{\prime}$ and the unprimed ones - to IRS K.

We begin with the formula (1). Let in IRS $K^{\prime}$ event 2 occurs after event 1 , so $t_{2}{ }^{\prime}>t_{1}{ }^{\prime}$ or $t_{2}{ }^{\prime}-t_{1}{ }^{\prime}>0$. Then for the inversion of time it is necessary that $t_{2}-t_{1}<0$, and then the second component of sum of the right-hand part of the formula (1) must be negative, so $x_{2}{ }^{\prime}-$ $x_{1}{ }^{\prime}<0$ and, in addition, the inequality must be carried out:

$$
\beta\left(x_{1}^{\prime}-x_{2}^{\prime}\right)>c\left(t_{2}^{\prime}-t_{1}^{\prime}\right)
$$

Let us consider the value $v$ with the dimension of velocity:

$$
v=\frac{x_{1}{ }^{\prime}-x_{2}{ }^{\prime}}{t_{2}{ }^{\prime}-t_{1}{ }^{\prime}}>\frac{c}{\beta}
$$

As you can see, this value must be greater than the velocity of light, which is impossible, and therefore inversion of time is only possible for the two events which are not connected by the cause-and-effect relation.

Now let us take formula (2). Let in IRS K event 2 occurs after event 1 , so $t_{2}>t_{1}$ or $t_{2}-t_{1}>0$. Then the inversion of time it is necessary $t_{2}{ }^{\prime}-t_{1}{ }^{\prime}<0$, and the second component of sum of formula (2) must be negative, so $x_{2}-x_{1}>0$ and, moreover, the inequality must be carried out:

$$
\beta\left(x_{2}-x_{1}\right)>c\left(t_{2}-t_{1}\right)
$$

Let as consider, as the previous case, the value $v$ with the dimension of speed: 


$$
v=\frac{x_{2}-x_{1}}{t_{2}-t_{1}}>\frac{c}{\beta}
$$

This value is again greater than the speed of light, and therefore the inversion of time is only possible for the two events which are not connected by the cause-and-effect relation.

Let us give the geometric interpretation of the phenomena from the point of view of Minkowski world. Let us consider the transition IRS K' $\rightarrow$ IRS K (Figure 2). In the initial IRS K' first event 1 occurs and then - event 2 (or 3 ) occurs. The case where the inequality (3) is not satisfied, corresponds to the point 2 . Here and in IRS K, as in the original IRS K' event 2 occurs after the event 1 , i.e. no inversion of time.

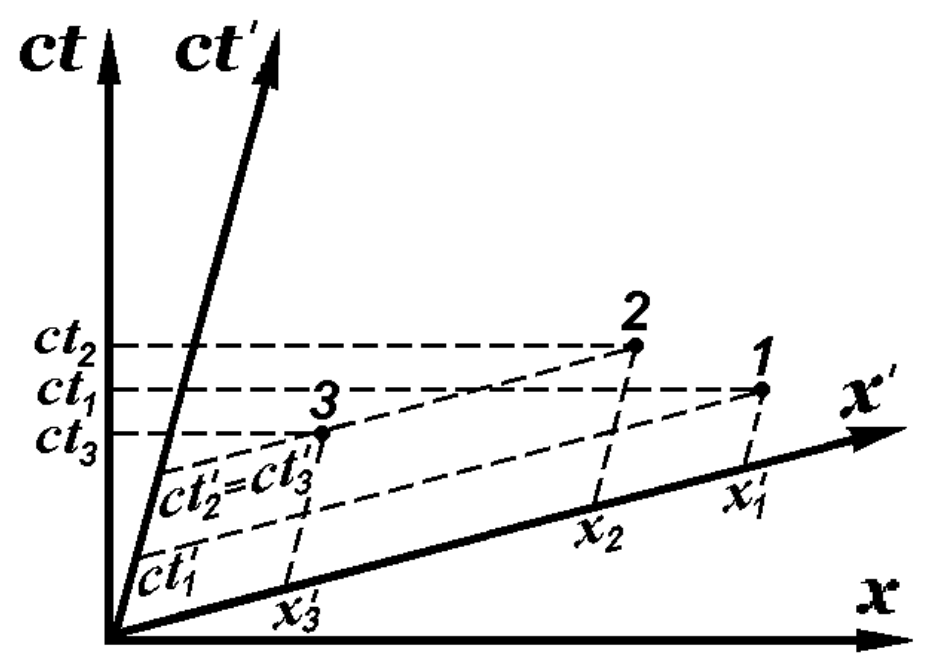

Fig. 2. The picture of inversion of time in Minkowski world.

The transition from IRS $K^{\prime}$ to IRS K

The case when the inequality (3) is satisfied corresponds to point 3. Here in IRS K event 3 occurs, as opposed to the IRS K', before event 1 , so the inversion of time occurs. (Or if the inequality (3) becomes the equality, the event 3 occurs simultaneously with event 1).

Now let us consider the transition IRS K $\rightarrow$ IRS K' (Figure 3). In the initial IRS K event 1 occurs first, and then event 2 (or 3 ) occurs. The case, when the inequality (5) is not satisfied, corresponds to the point 2 . Here in IRS K', as in the initial IRS K event 2 occurs after event 1 , so there is no inversion of time. 


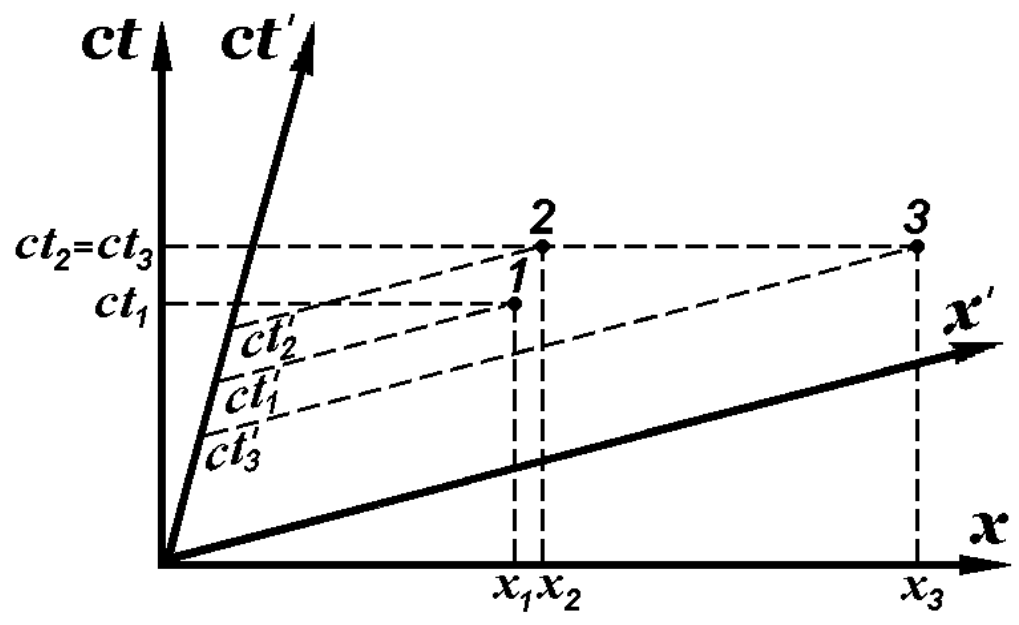

Fig. 3. The picture of inversion of time in Minkowski world.

The transition from IRS K to IRS K'.

The case, when the inequality (5) is satisfied, corresponds to the point 3. Here, in IRS K' event 3 occurs, unlike IRS K, before the event 1, i.e. there is the inversion of time. (Or, if inequality (5) becomes equation, the event 3 occurs simultaneously with event 1 ).

\section{Analysis of Results}

Thus, the conditions under which there is the inversion of time, or, in other words, mutually opposite directions of the flow of time for the two of IRS are connected with the following three factors:

1. Relative velocity of IRS.

2. Spatial distance between two points.

3. Period of time.

Let us consider all possible pairs of the IRS connected with real bodies of the Universe. In some of these pairs (and in some pairs of points) time flows in the same direction, and in some of them time flows in the opposite direction. Thus, the idea of the single direction of time in the Universe loses its sense. Past and future are relative. In the Universe temporal chaos reigns. The question of the age of the Universe loses sense too. Thus, we can say that the use of general relativity in cosmology, in a sense, contrary to the special relativity theory.

Of course, all these facts raise the question of the correspondence of the introduced concepts with the aggregate of the known experimental data in cosmology: the Big Bang and the age of the Universe, the expansion of the Universe, the relict radiation, dark matter and dark 
energy. We think all these phenomena can be interpreted in the context of new ideas, but some accents can be changed.

We can draw an analogy. Sometime in the XIX century, many of the phenomena of interpreted within the concept of the ether. And even when Maxwell's equations appeared, they were interpreted in the same way. Later when the concept of the ether dropped out, and all these phenomena remained.

We believe, it will turn out to be the same in this case: the concept of the single direction of time in the Universe will disappear and all observed phenomena will be treated in terms of the temporal chaos.

When special theory of relativity appeared, one of its unexpected and important results was the relativity of simultaneity. Now the elative of the direction of time for two events arises if they occur in different place and if they are not connected by cause-and-effect relation and formula (3) is performed for them.

Now let us estimate the order values describing different directions of time in the Universe. We use formula (3) in this case. The typical distance beginning from which the Universe can be considered homogeneous, and from which Friedman dynamic solution considered true about $100 \mathrm{Mps}$. Let us take $100 \mathrm{Mps}$ as the distance between two points in which two events occur. Let us the relative velocity of the two reference systems (for example, of two galaxies) be 0.1 light speed.

Then, for the inversion of time the interval between the two events in one reference system must not exceed 30 million years. Thus, from the point of view of the reference system which is connected with one of the galaxies, event 1 occurs 30 million years earlier than event 2 , then from point of view of the reference system which is connected with the other galaxy, event 1 occurs 30 million years later then event 2. Thus, we cannot speak of any arrow of time.

It is not difficult to select such parameters in the Universe under which inversion time difference exceeds the age of the Universe (13 billion years).

As it is known, the radius of the Universe is about $12000 \mathrm{Mps}$. Let us take $10000 \mathrm{Mps}$ as the distance between the two points and relative velocity of the reference systems (in which the two events occur) about the velocity of light. Then the difference of time is equal to 30 billion years. This value proves that the notion of the age of the Universe loses its sense.

We would like to stress that the given here calculations of the inversion of time are based on the special theory of relativity, and therefore they are obvious. Nevertheless, carrying out of an 
experiment confirming the inversion of time may be of some interest to us. The scheme of such an experiment could be next.

Let the primed IRS K' is connected with the Earth, and the unprimed IRS K - with two satellites, which have the zero velocity relative to each other and they are at some distance from each other. The clocks on these satellites are synchronized. Signals are sent from the satellites - at first from the one and then - from the other one. On Earth, these signals are received in reverse order.

Let us calculate the parameters of such an experiment. In order to get the inversion of time the second member of sum in equation (2) must be negative and grater in module than the first member of sum.

Take the velocity of the satellites relative to the Earth $V=10 \mathrm{Km} / \mathrm{s}=10^{4} \mathrm{~m} / \mathrm{s} \Delta x=x_{2}-$ $x_{1}=10^{3} \mathrm{Km}=10^{6} \mathrm{~m}-$ about $2,5 \%$ of the length Earth's equator.

Then the formula (5):

$$
\Delta t=t_{2}-t_{1}<\frac{\beta}{c}\left(x_{2}-x_{1}\right) \approx 10^{-7} \mathrm{~s}=100 \mathrm{~ns}
$$

Take $\Delta t=0,3 \cdot 10^{-7} \mathrm{~s}=30 \mathrm{~ns}$. Then the interval of time between the signals recorded on the Earth, is calculated by the formula (2) and its numerical value is $\Delta t^{\prime}=0,8 \cdot 10^{-7} \mathrm{~s}=$ 80 ns. The experiment seems to be feasible under these parameters nowadays.

\section{Spatial inversion}

Now let us consider the idea of spatial inversion in the framework of the transition from one IRS to another. As it is you known, in SRT there are the following formulas for the transformation of the coordinate difference.

Transition IRS K' $\rightarrow$ IRS K:

$$
\Delta x=x_{2}-x_{1}=\gamma\left[\left(x_{2}{ }^{\prime}-x_{1}{ }^{\prime}\right)+V\left(t_{2}{ }^{\prime}-t_{1}{ }^{\prime}\right)\right]
$$

Transition IRS K $\rightarrow$ IRS K':

$$
\Delta x^{\prime}=x_{2}{ }^{\prime}-x_{1}{ }^{\prime}=\gamma\left[\left(x_{2}-x_{1}\right)-V\left(t_{2}-t_{1}\right)\right]
$$


Let us begin our analysis with the transition IRS $K^{\prime} \rightarrow$ IRS K. Let in IRS K' event 1 occurs at the moment of time $t_{1}{ }^{\prime}$ at the point $x_{1}{ }^{\prime}$, and event 2 (or 3 ) - respectively at the moment of time $t_{2}{ }^{\prime}$ at the point $x_{2}{ }^{\prime}$ (or $t_{3}{ }^{\prime}$ and $x_{3}{ }^{\prime}$ ). Then, as it follows from formula (7) the condition of the spatial inversion is:

$$
t_{2}^{\prime}<t_{2}^{\prime} ; \quad V\left(t_{1}{ }^{\prime}-t_{2}{ }^{\prime}\right)>\left(x_{2}{ }^{\prime}-x_{1}{ }^{\prime}\right)
$$

According to the point of view of Minkowski world this picture is depicted in figure 4. There is now inversion at point 2 , and there is one at point 3.

Now let us consider the transition IRS K $\rightarrow$ IRS K'. As it follows from formula (8), the condition of spatial inversion has the form of:

$$
t_{2}>t_{1} ; \quad V\left(t_{2}-t_{1}\right)>\left(x_{2}-x_{1}\right)
$$

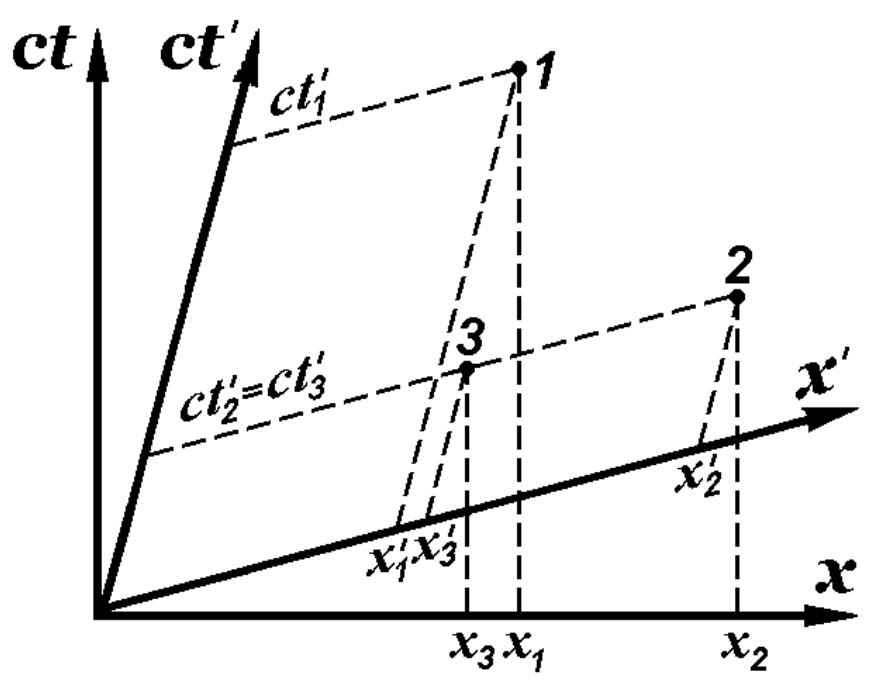

Fig. 4. The picture of spatial inversion in Minkowski world.

The transition from IRS $K^{\prime}$ to IRS K. 


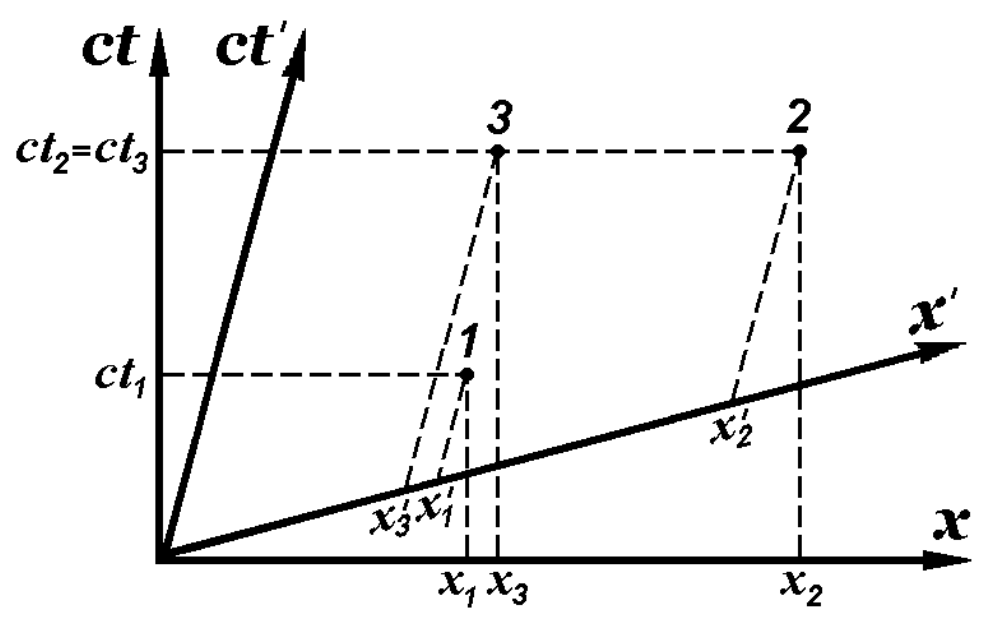

Fig. 5. The picture of spatial inversion in Minkowski world.

The transition from IRS K to IRS K'.

As before, the starting point - the point 1 and the final is point 2 (or 3 ). In accordance with Minkowski world this condition is depicted in figure 5. There is no spatial inversion at point 2, but there is one - at 3 point.

\section{Conclusions}

The main conclusions of this work are the following:

1. The inversion of time and spatial inversion (mirror reflection) to certain extent are equivalent to the transition from one IRS to another. Thus, it is possible to some extent to simulate inversion of time and mirror transformation.

2. In the Universe on the whole is no single direction of time. Let us call it the chaos of time of Universe.

Ad we believe this work can be developed further in the following directions:

1. The experimental search of the non-conservation parity in the processes caused by strong and electromagnetic interactions.

2. Development of methods for describing properties and behavior of big system (the Universe) in which time is chaotic but not dynamic. Static and dynamic views don't work. The views must be replaced by something else.

3. Coordination of the introduced here concepts with the aggregate of all known in cosmology experimental data. 
Proceedings of International Conference PIRT-2015

4. Analysis of the changes which must be made the proposed picture, taking into account the non-inertial of the real objects of the Universe and the gravitational effects.

5. Analysis of inversion effects in terms of three or more IRS. Multidimensional time is likely to appear.

6. Analysis of real scheme of the possible experiment (and its setting), which confirms the introduced here concepts.

\section{References}

1. Chelnokov M.B. (2015). Asymmetry Through the Looking Glass. Chebyshev’s sbornik, № 1, 281-290.

2. Ugarov V.A. (1977). Special Relativity Theory. Moscow: Science.

3. Fok V.A. (1955). Theory of space, time and gravitation. Moscow: GITTL. 